\title{
International Journal of
}

\section{Computer Network and Information Security}

\section{Kol.6}

No. 10 Sep. 2014

\section{IJCNIS Kol.6}

Http:// www.mecs-press .org Vol. 6 No.10 September 2014

\section{Modern Education}

and Computer Science PRESS 


\section{International Journal of Computer Network and Information Security (IJCNIS)}

ISSN Print: 2074-9090, ISSN Online: 2074-9104

Volume 6, Number 10, September 2014

\section{Contents}

\section{REGULAR PAPERS}

Hybrid Encryption-Compression Scheme Based on Multiple Parameter Discrete Fractional Fourier 1 Transform with Eigen Vector Decomposition Algorithm

Deepak Sharma, Rajiv Saxena, Narendra Singh

Distributed Monitoring for Wireless Sensor Networks: a Multi-Agent Approach

Bechar Rachid, Haffaf Hafid

Ternary Tree Based Group Key Agreement for Cognitive Radio MANETs

N. Renugadevi, C. Mala

Enhanced Intrusion Detection System for Malicious Node Detection in Mobile Ad hoc Networks using

Data Transmission Quality of Nodes

S. Mamatha, A. Damodaram

Efficient Clustering Technique for Cooperative Wireless Sensor Network

Kaushlendra Kumar Pandey, Neetesh Purohit, Ajay Agarwal

A Smart and Generic Secured Storage Model for Web based Systems

P.Iyappan, V.PrasannaVenkatesan

Hardware Realization of Fast Multi-Scalar Elliptic Curve Point Multiplication by Reducing the

Hamming Weights Over GF(p)

Nagaraja Shylashree, Venugopalachar Sridhar

Trust Metric based Soft Security in Mobile Pervasive Environment 